on a group or an individual level. Group experiential interventions focus on the infected worker.

Aim The purpose of such intervention programs will be the relief and emotional discharge of the symptoms of mobbing in workers in a hospital sector.

Material and methods In the focus groups will participate 40 health professionals voluntarily, in the intervention part of a study's protocol that is in process referring to a Doctoral Thesis in a Greek University. Sessions will take place in a public hospital, twice a week for 4 hours. 13 sessions will be held, organised in 4 groups of 10 people each. Interpersonal supportive psychotherapy treatment plan including techniques such as taking an individual-social-family history, role playing, drama therapy and cognitive behavioural therapy in a group therapeutic milieu. In particular, participants by playing roles, people relive the traumatic events, express their feelings, and the therapist helps to reduce negative emotions and to make adaptive beliefs. Also through the techniques of drama (mirror, chairs, narrative counselling, the individual expresses the history of his life in order to understand, evaluate and reassess his experiences.) The therapy does not aim at stirring internal psychological processes but illuminating the particular problem of a person-society relationship. The therapist's mission is to help the participants to overcome the problem. Through the techniques of cognitive behavioural therapy, participants will recognise their dysfunctional thoughts, process their feelings and behaviours about mobbing and with the help of the therapist will try to 'change' the way of thinking about their working lives.

Results This therapeutic approach will be used in order to strengthen the individuals self esteem, self appreciation and make them aware of their negative automatic cognitions and to produce data that may solve the problem of mobbing in their workplace.

Discussion The study expects to record accurately the size of the problem and indicate the appropriate tools for the implementation of interventions (prevention and awareness programs) both by mental health specialists and the decision makers and to enrich the scientific literature.

\section{EVALUATION OF EDUCATION OF SPECIALIST OCCUPATIONAL HEALTH PHYSICIANS IN THE PERIOD 2009-2013 IN FINLAND}

Jarmo Heikkinen*, Kimmo Räsänen. ,2Faculty of Health Sciences, Institute of Public Health and Clinical Nutrition, University of Eastern Finland, Kuopio campus

10.1136/oemed-2018-ICOHabstracts.287

Introduction A nationwide external quality evaluation aimed to identify strengths, good practices and areas in need of development.

Methods The enhancement-led four-phase evaluation process included a self-evaluation report by the universities; evaluation interviews and visits by the evaluators; an evaluation report; and a feedback seminar. The evaluation focused on the management and planning of education and the definition of learning outcomes and the learning philosophy; education resources and support and pedagogical training; the constructive alignment of education; and the continuous development of education.

Results Key strengths included good cooperation between units, pedagogical training of the trainers, investment in web- based learning and the use of previous evaluations in the development of education.

Changes in working life, the upcoming reform of specialist medical education and the continuity of funding were seen as external challenges.

Areas needing development included clarifying of the learning philosophy, broadening the learning outcomes to cover competences, achieving higher visibility, expanding and diversifying practices for learning assessment and feedback. Reflection practices were recommended to support interaction skills, professional growth and the promotion of development skills. Advancing pedagogical training of trainers was suggested. Genuinely interactive virtual learning was emphasised. Increasing the throughput of students and promoting interest in the specialty at the basic studies stage were recommended. The research-based and international approach in developing education was encouraged.

Discussion After the report the learning philosophy was clarified in the new curriculum, reflection was included in the logbook and a project to develop evaluation of the learning outcomes as competencies and entrustable professional activities started. A pedagogical web-based course and a pedagogical guide book for trainers were published. The specialty has worked vigorously on the external challenges, too.

High quality education is essential in occupational health care. An external evaluation of education may substantially help to meet the challenges of quality education.

\section{THE INTEGRATION OF OCCUPATIONAL HEALTH AND SAFETY INTO EDUCATION - ENETOSH'S WEALTH OF EXPERIENCE}

${ }^{1} \mathrm{U}$ Bollmann ${ }^{*},{ }^{2} \mathrm{R}$ Gründler, ${ }^{3} \mathrm{M}$ Holder. ${ }^{1}$ Institute for Work and Health of the German Social Accident Insurance (DGUV), Dresden, Germany; ${ }^{2}$ WissensImpuls, Dresden, Germany; ${ }^{3}$ British Safety Council (BSC), London, Great Britain

\subsection{6/oemed-2018-ICOHabstracts.288}

Introduction The results of an empirical study which analysed good-practice examples of integrating occupational health and safety into education are presented. The good practice was collected by the European Network Education and Training in Occupational Safety and Health (ENETOSH) over the last ten years and published on www.enetosh.net. The study was done in response to a request made by the International Labour Organisation (ILO) in order to make empirical statements on trends and promising models for integrating health and safety into education. Methods From 756 projects a representative sample of examples was chosen for each level of education (kindergarten/ school, initial vocational education and training, higher education and continuing vocational education and training). A category-system was developed which is based on the success principles of the settings-based approach as specified by the World Health Organisation (WHO). 83 examples were categorised and analysed. The study consists of a qualitative analysis as well as a statistical analysis. Within the qualitative part of the study interviews with project owners and leading experts in the field are carried out to gain experience of how to put the categories into practice.

Result The study provides a comprehensive evidence-base of practices of good models concerning mainstreaming occupational health and safety at all levels of education. The study facilitates better access to practices of good models for practitioners and 
policy makers and gives insight into the requirements of how to realise them. A report will be published end of the year 2017.

Discussion The study has identified gaps concerning the performance of empowerment and participation in the area of vocational education and training. There is a need for innovative learning environments especially for young workers and professionals to improve their health-competence.

\section{OCCUPATIONAL PSYCHOLOGY AND THERAPEUTIC YOGA -TOOLS FOR CRACKING HSE INTERNATIONAL CERTIFICATION EXAMS}

M Jayandran*. Green World Group Training and Consulting LLC, Chennai, India

\subsection{6/oemed-2018-ICOHabstracts.289}

Introduction Occupational Psychology is a Science of measuring psychological variables, such as knowledge, Self esteem, skills and abilities of an employee Understanding human behaviour in the workplace with respect to organisations principle and policies. A field that is concerned with the safety, physical and mental well being of an employee. Therapeutic Yoga - is an initiative which can be implemented by the Employer appointed competent Occupational yoga therapist and can be followed by the worker includes breath control, simple meditation, adoption of a specific bodily postures practiced for health and relaxation so as to perform well in the assigned tasks especially in high risk activities. It can be applied to maintain worker fitness in high risk activities like Work at Height and Confined Space and to remove their respective phobias i.e., Acrophobia and Claustrophobia and Mnemophobia for the aspirants who are undertaking International HSE competitive exams like NEBSOH, CSP etc.,. The Employee well being is a very important factor to control accidents in the workplace. Individual behaviour like Attitude, Competence, Perception, will play a vital role in Work Place Safety. This Individual behaviour and the mental well being can be highly improved if the employer applies the idea of Occupational Psychology and Therapeutic yoga.ILO 161 and ILO 1959 R112 defines 'occupational health services' states that safe and healthy working environment which will facilitate optimal physical and mental health in relation to work and the adaptation of work to the capabilities of workers Methods

a. Counselling Therapy: Counselling aims to help individual/

Employees to manage their accident prone psychological

difficulties and converting them to contribute for

Organisation Safety Occupational Psychologist has to

understand the types of psychological issues exist in the workplace and has to decide the counselling method as follows, Psychoanalytic therapy or talk therapy can be done by listening to Employees talk about their lives will release their personal stress.

b. Therapeutic Yoga blends postures, breath work, hands-on healing, meditation techniques which will improve the memory level for the aspirants who undergoing HSE training and preparing for competitive international HSE certification exams.

Results By applying counselling therapies and meditation stress control methods it has observed that the class test performance and the confidence level have improved very positively. Out of 6 aspirants, $60 \%$ improvement in terms of confidence and performance has achieved.
Conclusion The human mind and the individual factors are very challenging for the HSE Professionals. Continuous HSE trainings and knowledge updating is must for Safety Professionals. In between their work commitment, aspirants are emphasised by the organisation to take international HSE Certifications like IDIP NEBOSH, NEBOSH IGC, CSP. The aspirants greatly affected by mental stress with Mnemophobia. By adopting counselling therapies and therapeutic yoga to my aspirants, I found lot of improvement in their confidence as well as performance level. I recommend this method can be applied for other high risk activities like work at height, confined space to improve the physical and mental fitness of the workers.

Discussions Counselling aims to help individual/Employees to manage their accident prone psychological difficulties, builds confidence and converting them to contribute for Organisation Safety. Occupational Psychologist has to understand the types of psychological issues exist in the workplace and has to decide the counselling method as follows, Psychoanalytic therapy or talk therapy can be done by listening to Employees talk about their lives will release their personal stress. In Behavioural therapy, employees encouraged to gradually face their fear of heights by explaining about the safety precautions adapted in Work at heights. Group therapy is a form of psychotherapy where two or more employees will insist to play a game with their superiors will pave the way healthy dignified relationship. Art therapy involves the use of artistic means to work through difficult emotions. It helps individuals who are having trouble discussing their problems verbally. in the same way, therapeutic yoga postures will boost the mental as well as, physical fitness.

Being a HSE Tutor handling HSE Professionals of different industrial background and tutoring them to take up international HSE certifications like IOSH MS, NEBOSH IGC etc., I identified they are undergoing severe stress and psychological issues due to so many reasons. So, I applied this concept of Counselling therapies, therapeutic yoga and found improvement as follows: Absenteeism rate reduced, Motivation and self confidence increased, Considerable amount of optimistic changes observed in Daily test performance. I suggest, the safety professionals has to implement this techniques to improve the resilience, fitness of the employees so as to create good safety culture and competent work group for high hazardous activities like work at height, confined space safety.

\section{THE EFFECTS OF TRAINING MANAGERS ON MANAGEMENT COMPETENCIES ON WORK ENGAGEMENT OF THEIR SUBORDINATES: A SINGLE- GROUP PRE- AND POST-TEST STUDY}

Y Sekiya*, H Adachi, K Imamura, K Watanabe, N Kawakami. Department of Mental Health, Graduate School of Medicine, The University of Tokyo, Tokyo, Japan

\subsection{6/oemed-2018-ICOHabstracts.290}

Introduction The UK Health and Safety Executive (HSE) developed the concept and measure called 'Stress management competency indicator tool' of management competencies to prevent and reduce stress at work, and has shown some beneficial effect of training managers for these competencies (Yarker, et al, 2008). The purpose of this study was to examine the effect of a training program for managers developed 